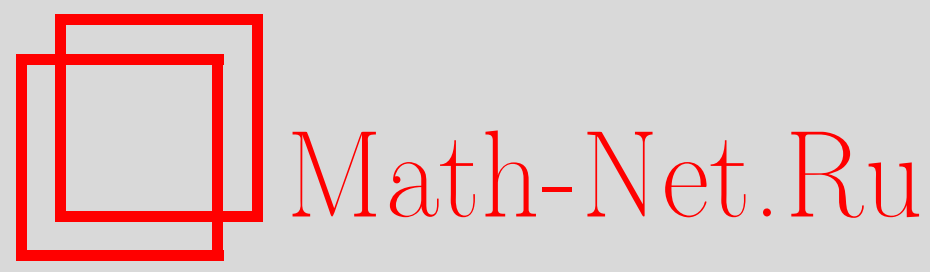

М. И. Тихомирова, В. П. Чистяков, О свойствах функции правдоподобия в задаче оценки моментов изменения вероятности успеха в неоднородных испытаниях Бернулли, Дискрет. матем., 2010, том 22, выпуск 2, 60-65

DOI: https://doi.org/10.4213/dm1095

Использование Общероссийского математического портала Math-Net.Ru подразумевает, что вы прочитали и согласны с пользовательским соглашением http://www . mathnet.ru/rus/agreement

Параметры загрузки:

IP : 54.237 .59 .107

26 апреля 2023 г., 18:08:56 


\title{
О свойствах функции правдоподобия в задаче оценки моментов изменения вероятности успеха в неоднородных испытаниях Бернулли
}

\author{
() 2010 г. М. И. Тихомирова, В. П. Чистяков
}

\begin{abstract}
Получено явное выражение для статистики, являющейся линейной функцией от логарифма функции правдоподобия, исследуются свойства ее математического ожидания. Полученные результаты могут быть применены к оцениванию моментов многократных изменений вероятности успеха.

Работа выполнена при поддержке программы президента Российской Федерации поддержки ведущих научных школ, проект НШ 4129.2006.1
\end{abstract}

\section{1. Введение}

Задачи обнаружения моментов изменения вероятностных характеристик случайных последовательностей (задачи разладки) рассматривались многими авторами (см., например, [1-6]). Сложилось два направления исследований: скорейшее обнаружение в текущем контроле очередного момента изменения вероятностных характеристик случайной последовательности, обнаружение всех моментов смены вероятностных характеристик в заданном отрезке случайной последовательности. Данная работа относится ко второму направлению.

Пусть

$$
x_{1}, x_{2}, \ldots, x_{t}, \ldots
$$

- последовательность независимых случайных величин, каждая из которых принимает два значения 0 или 1 (успех или неудача). Вероятности этих значений зависят от $t$ :

$$
\mathbf{P}\left\{x_{t}=1\right\}=p_{i}, \quad t \in\left\{t_{i-1}+1, \ldots, t_{i}\right\}, \quad i=1,2, \ldots,
$$

где $0<p_{i}<1, p_{i} \neq p_{i+1}, 0=t_{0}<t_{1}<\ldots<t_{r}<\ldots$ Будем предполагать, что вероятности $p_{i}$ известны и известно, что в $T$ первых членах последовательности

$$
x_{1}, x_{2}, \ldots, x_{T}, \quad t_{r}<T \leqslant t_{r+1},
$$

произошло ровно $r$ изменений вероятности успеха, моменты $t_{1}, \ldots, t_{r}$ предполагаются неизвестными. В работе исследуется математическое ожидание линейной функции от логарифма функции правдоподобия. Показано, как установленные свойства этого математического ожидания могут быть использованы для оценивания моментов смены вероятности успеха. 


\section{2. Функция правдоподобия}

Не ограничивая общности, можно положить $T=t_{r+1}$. Тогда функция правдоподобия принимает вид

$$
L\left(x_{1}, \ldots, x_{T}\right)=\prod_{i=1}^{r+1} \prod_{j=t_{i-1}+1}^{t_{i}} p_{i}^{x_{t_{j}}} q_{i}^{\bar{x}_{t_{j}}}
$$

где $\bar{x}_{s}=1-x_{s}, q_{i}=1-p_{i}$. Отсюда, полагая

$$
\mu\left(t_{i-1}, t_{i}\right)=\sum_{s=t_{i-1}+1}^{t_{i}} x_{s}
$$

получим равенство

$$
\ln L\left(x_{1}, \ldots, x_{T}\right)=\sum_{i=1}^{r+1}\left[\mu\left(t_{i-1}, t_{i}\right) \ln p_{i}+\left(t_{i}-t_{i-1}\right) \mu\left(t_{i-1}, t_{i}\right) \ln q_{i}\right]
$$

Число $\mu(u, v)$ единиц в множестве $\left\{x_{u+1}, \ldots, x_{v}\right\}$ выразим через числа $\mu_{s}=\mu(0, s)$ единиц в множествах $\left\{x_{1}, \ldots, x_{s}\right\}: \mu(u, v)=\mu_{v}-\mu_{u}$. Используя эти обозначения, запишем (4) в следующем виде:

$$
\ln L\left(x_{1}, \ldots, x_{T}\right)=\sum_{i=1}^{r} \mu_{t_{i}} a_{i}+\mu_{T} \ln \frac{p_{r+1}}{q_{r+1}}+T \ln q_{r+1}+\sum_{i=1}^{r} t_{i} b_{i},
$$

где

$$
a_{i}=\ln \frac{p_{i} q_{i+1}}{q_{i} p_{i+1}}, \quad b_{i}=\ln \frac{q_{i}}{q_{i+1}}, \quad i=1, \ldots, r .
$$

Логарифм функции правдоподобия (5) будем рассматривать как функцию $\tilde{\eta}_{T}\left(t_{1}, \ldots, t_{r}\right)$ от неизвестных точек $\left(t_{1}, \ldots, t_{r}\right)$ изменения вероятностей успеха. Отбросив в (5) слагаемые, не зависящие от $t_{1}, \ldots, t_{r}$, и заменив $t_{1}, \ldots, t_{r}$ переменными $s_{1}, \ldots, s_{r}$, получим статистику

$$
\eta_{T}\left(s_{1}, \ldots, s_{r}\right)=\sum_{i=1}^{r} f_{i}^{*}\left(s_{i}\right), \quad f_{i}^{*}(s)=\mu_{s} a_{i}+s b_{i}
$$

где $a_{i}$ и $b_{i}$ определены равенствами (6). Очевидно, что у функций $\tilde{\eta}_{T}\left(s_{1}, \ldots, s_{r}\right)$ и $\eta_{T}\left(s_{1}, \ldots, s_{r}\right)$ точки, в которых они имеют локальные максимумы и минимумы, совпадают.

Найдем $\varphi_{T}\left(s_{1}, \ldots, s_{r}\right)=\mathbf{E} \eta_{T}\left(s_{1}, \ldots, s_{r}\right)$. Для этого воспользуемся формулой для математического ожидания $g(s)=\mathbf{E} \mu_{s}$ числа единиц в $s$ первых членах последовательности (1):

$$
g(s)= \begin{cases}p_{1} s, & 1 \leqslant s \leqslant t_{1}, \\ \sum_{i=1}^{j-1} p_{i}\left(t_{i}-t_{i-1}\right)+p_{j}\left(s-t_{j-1}\right), & t_{j-1} \leqslant s \leqslant t_{j}, j=2, \ldots, r+1 .\end{cases}
$$


Теорема 1. Математическое ожидание

$$
\mathbf{E} \eta_{T}\left(s_{1}, \ldots, s_{r}\right)=\varphi_{T}\left(s_{1}, \ldots, s_{r}\right)
$$

можно представить в виде

$$
\varphi_{T}\left(s_{1}, \ldots, s_{r}\right)=\sum_{i=1}^{r} f_{i}\left(s_{i}\right),
$$

где

$$
\begin{aligned}
f_{i}(s) & =\mathbf{E} f_{i}^{*}(s) \\
& = \begin{cases}c_{i 1} s, & 1 \leqslant s \leqslant t_{1}, \\
\sum_{l=1}^{j} c_{i l}\left(t_{l}-t_{l-1}\right)+c_{i, j+1}\left(s-t_{j}\right), & t_{j} \leqslant s \leqslant t_{j+1}, j=1, \ldots, r,\end{cases} \\
c_{i l} & =p_{l} \ln \frac{p_{i}\left(1-p_{i}\right)^{1 / p_{l}-1}}{p_{i+1}\left(1-p_{i+1}\right)^{1 / p_{l}-1}} .
\end{aligned}
$$

При любых $p_{i} \neq p_{i+1}$ верны неравенства

$$
c_{i i}>0, \quad c_{i, i+1}<0, \quad i=1, \ldots, r .
$$

Доказательство. Формулы (10) и (11) следуют из формул (7), (8) и из равенства

$$
\mathbf{E}\left(\mu_{s} a_{i}+s b_{i}\right)=g(s) a_{i}+s b_{i},
$$

в котором нужно заменить $g(s)$ выражением (8), а $s$ в произведении $s b_{i}$ представить в виде

$$
s=\left(s-t_{j-1}\right)+\sum_{k=1}^{j-1}\left(t_{k}-t_{k-1}\right) .
$$

Неравенства (12) следуют из неравенств

$$
h(x)<h\left(x_{\alpha}\right), \quad x \in\left(0, x_{\alpha}\right) \cup\left(x_{\alpha}, 1\right),
$$

где

$$
h(x)=x(1-x)^{\alpha}, \quad x_{\alpha}=\frac{1}{1+\alpha}, \quad \alpha>0 .
$$

При $x=p_{i+1}, \alpha=1 / p_{i}-1$ получаем первое неравенство (12), а при $x=p_{i}$, $\alpha=1 / p_{i+1}-1$ получаем второе. Теорема доказана.

Отметим некоторые свойства функций $f_{i}(s), i=1, \ldots, r$, которые следуют из теоремы 1.

(1) График функции $f_{i}(s), i=1, \ldots, r$, является ломаной, а угол при ее вершине $\left(t_{i}, f_{i}\left(t_{i}\right)\right)$ образован двумя прямыми с угловыми коэффициентами $c_{i i}>0$ и $c_{i, i+1}<0$.

(2) Функция $f_{i}(s), i=1, \ldots, r$, на отрезке $\left[t_{i-1}, t_{i+1}\right]$ имеет единственный максимум в точке $t_{i}$. 
(3) При любых $p_{i} \neq p_{i+1}, i=1, \ldots, r$, функция $\varphi_{T}\left(s_{1}, \ldots, s_{r}\right)$ имеет единственный максимум на множестве

$$
S=\left\{\left(s_{1}, \ldots, s_{r}\right): t_{i-1} \leqslant s_{i} \leqslant t_{i+1}, i=1, \ldots, r\right\}, \quad 0=t_{0}<t_{1} \ldots<t_{r+1}=T,
$$

и

$$
\max _{\bar{s} \in S} \varphi_{T}\left(s_{1}, \ldots, s_{r}\right)=\sum_{i=1}^{r} \max _{t_{i-1} \leqslant s \leqslant t_{i+1}} f_{i}(s) .
$$

(4) При $i \geqslant 2$ первый локальный максимум функции $f_{i}(s)$ на множестве $s \geqslant t_{k-1}$ достигается в точке $s=t_{k}$.

Рассмотрим более простой случай двух чередующихся вероятностей успеха:

$$
p_{2 u+1}=p_{1}, \quad p_{2(u+1)}=p_{2}, \quad u=0,1, \ldots, \quad p_{1} \neq p_{2} .
$$

В этом случае

$$
f_{2 u+1}(s)=f_{1}(s), \quad f_{2 u+2}(s)=-f_{1}(s) .
$$

Локальные максимумы функции $f_{1}(s)$ достигаются в точках $t_{1}, t_{3}, \ldots$, а локальные максимумы функции $f_{2}(s)=-f_{1}(s)-$ в точках $t_{2}, t_{4}, \ldots$ Следовательно, функция $\varphi_{T}\left(s_{1}, \ldots, s_{r}\right)$ имеет локальный максимум только в точках $\left(s_{1}^{(0)}, \ldots, s_{r}^{(0)}\right)$, где $s_{i}^{(0)} \in\left\{t_{1}, t_{3}, \ldots\right\}$ при нечетном $i$ и $s_{i}^{(0)} \in\left\{t_{2}, t_{4}, \ldots\right\}$ при четном $i$. Точка $\left(t_{1}, \ldots, t_{r}\right)$ истинных моментов смены вероятностей успеха является точкой локального максимума функции $\varphi_{T}\left(s_{1}, \ldots, s_{r}\right)$ с координатами $s_{1}^{(0)}, \ldots, s_{r}^{(0)}$, удовлетворяющими условию $s_{1}^{(0)}<s_{2}^{(0)}<\ldots<s_{r}^{(0)}$.

Таким образом, для определения локального максимума функции $\varphi_{T}\left(s_{1}, \ldots, s_{r}\right)$ от $r$ переменных в этом случае достаточно найти локальные максимумы двух функций от одной переменной.

\section{3. Оценивание моментов изменения вероятности успеха}

Приведенные в параграфе 2 свойства позволяют предложить различные процедуры получения оценок $\bar{t}^{*}=\left(t_{1}^{*}, \ldots, t_{r}^{*}\right)$ истинной точки $\bar{t}=\left(t_{1}, \ldots, t_{r}\right)$ смены вероятностей успеха.

Процедура 1. Используя свойство 4, можно предложить следующую процедуру получения оценки $\bar{t}^{*}$. Сначала по функции $f_{1}^{*}(s)$ определить точку $t_{1}^{*}$, соответствующую первому локальному максимуму функции $f_{1}(s)$, затем при $s>t_{1}^{*}$ по функции $f_{2}^{*}(s)$ выбрать точку, соответствующую $t_{2}$, и так далее.

В общем случае при определении оценок максимального правдоподобия $r$ параметров требуется определить точку, в которой достигается максимум функции $r$ переменных. В предложенной выше процедуре нужно искать максимум $r$ функций одной переменной.

Процедура 2. Если из дополнительной информации удалось найти множество

$$
S^{*}=\left\{\left(s_{1} \ldots, s_{r}\right): t_{i-1}+\underline{s}_{i}<s_{i}<t_{i+1}-\bar{s}_{i}, i=1, \ldots, r\right\} \subset S,
$$


где числа $\underline{s}_{i}, \bar{s}_{i}$ удовлетворяют условиям $t_{i-1}+\underline{s}_{i}<t_{i}<t_{i+1}-\bar{s}_{i}, \underline{s}_{i}>0, \bar{s}_{i}>0$, $i=1, \ldots, r$, то оценку $\bar{t}^{*}=\left(t_{1}^{*}, \ldots, t_{r}^{*}\right)$ точки $\bar{t}=\left(t_{1}, \ldots, t_{r}\right)$ можно определить равенствами

$$
\max _{t_{i_{1}}+\underline{s}_{i} \leqslant s_{i} \leqslant t_{i+1}-\bar{s}_{i}} f_{i}^{*}\left(s_{i}\right)=f_{i}^{*}\left(t_{i}^{*}\right), \quad i=1, \ldots, r
$$

где функция $f_{i}^{*}(s)$ определена вторым равенством (7). Если при данном $i$ точек $t_{i}^{*}$ несколько, то выбирается наименьшая из них.

На множестве $S^{*}$ по свойству 3 функция $\varphi_{T}\left(s_{1}, \ldots, s_{R}\right)$ имеет единственный максимум. Из свойства 2 и закона больших чисел следует утверждение следующей теоремы 2.

Теорема 2. Если $p_{i} \neq p_{i+1} i=1, \ldots, r-1$ и при $T \rightarrow \infty$ точки $\bar{t}(T)=\left(t_{1}, \ldots, t_{r}\right)$, $\bar{s}(T)=\left(s_{1}, \ldots, s_{r}\right)$ меняются так, что $t_{l} / T \rightarrow \gamma_{l}, s_{l} / T \rightarrow \beta_{l}, 0<\gamma_{l}<\infty, 0<\beta_{l}<\infty$, $\gamma_{l-1}<\beta_{l}<\gamma_{l+1}, l=1, \ldots, r, \gamma_{r+1}=1$, то для любого $\varepsilon \in(0,1)$ найдется такое $T_{0}$, что вероятность события $\eta_{T}\left(s_{1}, \ldots, s_{r}\right)<\eta_{T}\left(t_{1}, \ldots, t_{r}\right)$ больше $1-\varepsilon$ при $T>T_{0}$.

При отсутствии дополнительной информации множество $S^{*}$ можно выбрать, воспользовавшись для предварительной оценки точки $t=\left(t_{1}, \ldots, t_{r}\right)$ статистиками

$$
\xi_{T}^{(h)}(s)=\frac{1}{\sqrt{T}}\left(\mu_{s+h}-2 \mu_{s}+\mu_{s-h}\right), \quad s=h+1, \ldots, T-h, \quad h>0 .
$$

Абсолютная величина математического ожидания этих статистик $\left|\mathbf{E} \xi_{T}^{(h)}(s)\right|$, рассматриваемая как функция одной переменной $s$, имеет локальные максимумы в точках $t_{1}, \ldots, t_{r}$.

Приведем без доказательства теорему о предельных распределениях для статистик (18) в схеме серий при $T \rightarrow \infty$, когда $p_{k}=p_{k}(T), h=h(T)$ и $t_{k}=t_{k}(h)$ изменяются следующим образом:

$$
\begin{aligned}
p_{k}(T) & =\left(1+\frac{\omega_{k}(T)}{\sqrt{T}}\right) p, & 0<p<1, \\
\lim _{T \rightarrow \infty} \omega_{k}(T) & =\omega_{k}, \quad \lim _{t \rightarrow \infty} \frac{h(T)}{T}=h_{0}, \quad \lim _{t \rightarrow \infty} \frac{t_{k}(T)}{T}=\gamma_{k}, & k=1, \ldots, r .
\end{aligned}
$$

Обозначим через $\zeta(\theta), 0 \leqslant \theta \leqslant 1$, гауссовский процесс с математическими ожиданиями

$$
\mathbf{E} \zeta(\theta)= \begin{cases}p\left(\omega_{l+1}-\omega_{l}\right)\left(h_{0}-\left|\theta-\gamma_{l}\right|\right), & \gamma_{l}-h_{0}<\theta<\gamma_{l}+h_{0}, l=1, \ldots, r, \\ 0 & \text { в остальных случаях, }\end{cases}
$$

и ковариационной матрицей

$$
R\left(\theta^{\prime}, \theta^{\prime \prime}\right)= \begin{cases}p(1-p)\left(2 h_{0}-\left|\theta^{\prime}-\theta^{\prime \prime}\right|\right), & \left|\theta^{\prime}-\theta^{\prime \prime}\right| \leqslant 2 h_{0} \\ 0 & \text { в остальных случаях. }\end{cases}
$$

Теорема 3. Если $\theta_{i}^{*}=s_{i} / T \rightarrow \theta_{i}, i=1, \ldots, m$, при $T \rightarrow \infty$, то в схеме серий (19) совместные распределения величин $\xi_{T}^{(h)}\left(\theta_{i}^{*}\right), i=1, \ldots, m$, сходятся к совместному распределению величин $\zeta\left(\theta_{i}\right), i=1, \ldots, m$.

Отметим, что функция $|\mathbf{E} \zeta(\theta)|$ имеет локальные максимумы в точках $\theta_{l}=\gamma_{l}$, $l=1, \ldots, r$, соответствующих точкам смены вероятностей $p_{l}$ на $p_{l+1}$. Таким образом, последовательность статистик (18) может быть использована для предварительной оценки $\left(t_{1}, \ldots, t_{r}\right)$. 
Таблица 1. $T=3900, p_{2}=0,7, p_{1}=p_{3}, t_{1}=1300, t_{2}=2600, b=1000, H=30$

\begin{tabular}{cccrrccc}
\hline$p_{1}$ & $\hat{m}_{t_{1}}$ & $\hat{m}_{t_{2}}$ & $\hat{\sigma}_{t_{1}}$ & $\hat{\sigma}_{t_{2}}$ & $\hat{p}_{1}$ & $\hat{p}_{2}$ & $\hat{p}_{12}$ \\
\hline 0,3 & 1298,4 & 2538,5 & 7,52 & 6,79 & 0,989 & 0,991 & 0,98 \\
0,4 & 1299,6 & 2599,9 & 13,85 & 13,31 & 0,947 & 0,958 & 0,905 \\
0,5 & 1300,6 & 2599,8 & 31,39 & 29,37 & 0,793 & 0,812 & 0,647 \\
0,6 & 1298,8 & 2598,4 & 97,30 & 99,05 & 0,446 & 0,473 & 0,206 \\
\hline
\end{tabular}

Таблица 2. $T=300, p_{2}=0,7, p_{1}=p_{3}, t_{1}=100, t_{2}=200, b=1000, H=30$

\begin{tabular}{rrrrrrcc}
\hline$p_{1}$ & \multicolumn{1}{c}{$\hat{m}_{t_{1}}$} & $\hat{m}_{t_{2}}$ & \multicolumn{1}{c}{$\hat{\sigma}_{t_{1}}$} & $\hat{\sigma}_{t_{2}}$ & $\hat{p}_{1}$ & $\hat{p}_{2}$ & $\hat{p}_{12}$ \\
\hline 0,3 & 98,72 & 199,00 & 6,98 & 7,37 & 0,992 & 0,991 & 0,983 \\
0,4 & 99,74 & 200,91 & 11,295 & 12,55 & 0,962 & 0,955 & 0,918 \\
0,5 & 100,22 & 201,73 & 22,74 & 22,26 & 0,8279 & 0,843 & 0,701 \\
0,6 & 98,92 & 201,36 & 37,68 & 38,55 & 0,590 & 0,578 & 0,350 \\
\hline
\end{tabular}

Некоторое представление о точности оценок $\left(t_{1}^{*}, \ldots, t_{r}^{*}\right)$ моментов $\left(t_{1}, \ldots, t_{r}\right)$ дает обработка реализаций последовательности (1), полученных с использованием датчика случайных чисел. При $r=2$ было получено по $n$ реализаций $\left(x_{1}^{(l)}, \ldots, x_{T}^{(l)}\right), l=1, \ldots, n$, при различных наборах значений параметров $T, p_{1}, p_{2}, t_{1}$ и $t_{2}$. Предполагается, что числа $\bar{s}_{l}$ и $\underline{s}_{l}, l=1,2$, в равенстве (17) известны. Для каждой реализации с использованием (17) были получены оценки $\left(t_{1}^{*}(l), t_{2}^{*}(l)\right), l=1, \ldots, n$. Оценками $m_{t_{l}}=\mathbf{E} t_{l}^{*}, \sigma_{t_{l}}^{2}=\mathbf{D} t_{l}^{*}$ являются выборочные математические ожидания и дисперсии $\hat{m}_{t_{l}}, \hat{\sigma}_{t_{l}}^{2}$. Вероятности $P_{l}=$ $\mathbf{P}\left\{t_{l}^{*} \in\left(t_{l}-H, t_{l}+H\right), l=1,2\right\}, P_{12}=\mathbf{P}\left\{\left(t_{1}^{*}, t_{2}^{*}\right) \in\left(t_{1}-H, t_{1}+H\right) \times\left(t_{2}-H, t_{2}+H\right)\right\}$ оценивались частотами $\hat{p}_{l}$ и $\hat{p}_{12}$ соответствующих событий. Полученные результаты приведены в табл. 1 и 2.

Приведенные в таблицах оценки математических ожиданий $\mathbf{E} t_{1}^{*}, \mathbf{E} t_{2}^{*}$ близки к истинным моментам разладки $\left(t_{1}, t_{2}\right)$ при всех рассмотренных значениях параметров. Качество оценок характеризуется значением среднеквадратичных отклонений $\hat{\sigma}_{t_{k}}$ и частотами $\hat{p}_{l}$, $\hat{p}_{12}$. По результатам эксперимента точность оценок уменьшается при сближении вероятностей $p_{1}$ и $p_{2}$.

\section{Список литературы}

1. Боровков А. А., Асимптотически оптимальные решения в задаче о разладке. Теория вероятностей и ее применения (1998) 43, №4, 623-654.

2. Дарховский Б. С., Непараметрический метод оценивания интервалов однородности случайной последовательности. Теория вероятностей и ее применения (1985) 30, №4, 795-799.

3. Hinkley D. V., Hinkley E. A., Inference about the change-point in a sequence of binomial variables. Biometrika (1970) 57, 477-488.

4. Хахубия Ц. Г., Предельная теорема для оценки максимального правдоподобия момента разладки. Теория вероятностей и ее применения (1986) 31, №1, 152-155.

5. Zubkov A. M., Sokolov D. V., Statistical inference for noisy piecewise linear signals.. В сб.: Докл. 1-й Межд. конф. “Цифровая обработка сигналов и ее применения”, II-E, Москва, 1998, с. 140-142.

6. Бродский Б. Е., Дарховский Б. С., Проблемы и методы вероятностной диагностики. Автоматика и телемеханика (1999) 8, 3-50. 